\title{
Human milk: composition and health benefits
}

\author{
Fabio Mosca, Maria Lorella Giannì \\ Neonatal Intensive Care Unit, Department of Clinical Science and Community Health, IRCCS Foundation Ca' Granda \\ Ospedale Maggiore Policlinico, University of Milan, Milan, Italy
}

\begin{abstract}
Breastfeeding is widely acknowledged as the normal and unequalled method for feeding infants due to its associated health benefits, both for the infant and the mother. The World Health Organization recommends that infants are exclusively breastfed up to the completion of six months of age, with breastfeeding continuing to be an important part of the diet until the infant is at least two years old. The several health benefits associated with breastfeeding are driven by the combined action of the nutritional and bioactive components in human milk and the magnitude of the majority of the ascertained biological effects is directly dependent on breastfeeding duration. This review briefly summarizes the current knowledge on the composition of human milk and provides an overview on its functional effects on health outcomes, focusing on the latest research results.
\end{abstract}

\section{Introduction}

Extensive evidence indicates that breastfeeding is essential to ensure healthy growth and development of infants and children. ${ }^{1}$

Correspondence: Maria Lorella Giannì, Neonatal Intensive Care Unit, IRCCS Foundation Ca' Granda Ospedale Maggiore Policlinico, University of Milan, via Commenda 12, Milano 20122, Italy.

Phone +39.02.55032483 - Fax +39.02.55032436.

Email: maria.gianni@unimi.it

Key words: Human milk; Composition; Benefits; Infant.

Contributions: the authors contributed equally.

Conflict of interest: the authors declare no potential conflict of interest.

Funding: Funded by Merqurio Editore S.r.l., with the unconditional contribution of Nestlè Italiana S.p.A.

Received for publication: 7 June 2017.

Accepted for publication: 20 June 2017.

This work is licensed under a Creative Commons Attribution NonCommercial 4.0 License (CC BY-NC 4.0).

(C) Copyright F. Mosca and M.L. Giannì, 2017

Licensee PAGEPress, Italy

La Pediatria Medica e Chirurgica 2017; 39:155

doi:10.4081/pmc.2017.155
The World Health Organization recommends that infants are exclusively breastfed up to the completion of six months, with breastfeeding continuing to be an important part of the diet until the infant is at least two years old. ${ }^{2}$

Human milk is an individual-specific biofluid, characterized by an extreme variability in its composition, with regard to both nutritional and bioactive components. From an evolutionary perspective, its composition has evolved over time in order to provide the infant a well-balanced nutrition and protection against potential infectious pathogens while the neonatal immune system completes its development. ${ }^{3}$ Influences on compositional differences include time of lactation, length of gestation, maternal diseases, genotype and diet. ${ }^{4}$ The mean protein content gradually decreases from the second to the sixth-seventh month of lactation and stabilizes thereafter. ${ }^{4,5}$ Remarkably, preterm milk content through early lactation is higher than that of term milk (Figure 1). ${ }^{5}$

Lactose content is particularly high between the fourth and the seventh month of lactation and decreases afterwards. On the contrary, fat content increases throughout lactation. Fat content varies according to the stage of nursing process, being most represented at the end of a feed, and to the time of the day. ${ }^{6}$ The fat fraction appears to be the most sensible to maternal diet whereas the protein and carbohydrate fraction appear not to be affected. ${ }^{7}$ The specific fatty acids in human milk, either synthetized endogenously in the mammary gland or taken up from maternal plasma, reflect changes in the maternal dietary fat within two-three days. Maternal body mass index also contributes to modulate the amount and type of human milk fatty acids; specifically, the amount of saturated fatty acids and the ratio of n- 6 to n- 3 fatty acids are higher in overweight women's breast milk than in normal weight women's breast milk, also after taking into account maternal diet. 8

This review briefly summarizes the current knowledge on the composition of human milk and provides an overview on its functional effects on health outcomes, focusing on the latest research results.

\section{Stages of lactation}

Human milk is commonly distinct into colostrum, transitional and mature milk. However, it has to be taken into account that this classification refers to the gradual changes that occur in human milk according to stage of lactation rather than indicating specific classes of milk. Colostrum, the first milk produced, contains a high concentration of whey proteins, with casein being almost undetectable, whereas the lactose and fat content is lower compared to that in mature milk. 4,9 One of the main feature of colostrum is represented by the high concentration in bioactive compounds, including secretory immunoglobulin (IgA), lactoferrin and leukocytes. ${ }^{10}$ The high content of secretory immunoglobulins indicates that the main role 
of colostrum is immunologic rather than nutritional. The protective function of colostrum is further underlined by the particularly high content of oligosaccharides, that approximately doubles that of mature milk, decreasing from $21 \mathrm{~g} / 100 \mathrm{~mL}$ on the $4^{\text {th }}$ day to $13 \mathrm{~g} / 100$ $\mathrm{mL}$ at the end of the second month of lactation. ${ }^{11}$ On the basis of the studies focusing on human milk composition, it appears that colostrum may also act as growth promoter, since it contains high concentrations of epidermal growth factor, TGF- $\beta$ and colony stimulating factor. ${ }^{10}$ After 5 days, colostrum will be gradually replaced by transitional milk which shares some features of colostrum and support infants' growth and development. From the second week after delivery, milk is considered largely mature, becoming fully mature by the $4^{\text {th }}$ to the $6^{\text {th }}$ week. ${ }^{9}$

\section{Composition}

\section{Proteins}

Proteins represent the third abundant solids in human milk, providing not only nutrition but also performing several bioactive functions. Indeed, proteins are essential to allow for infants' healthy growth but they act also as carriers for other nutrients (i.e., lactoferrin, haptocorrin, alfa-lactalbumin, folate-binding protein, beta-casein), promote gut development (growth factors, lactoferrin) and nutrients absorption (bile salt-stimulated lipase, amylase, alfa1-antitrypsin), and possess immune and antimicrobial activity (lactoferrin, secretory IgA, osteopontin, cytokines, lysozyme, etc.). ${ }^{12}$ Milk proteins can be grouped into three major classes: caseins, whey and mucins. Caseins are assembled in micelles whereas whey proteins are present in solution. The main whey proteins are represented by alpha-lactalbumin, lactoferrin, lysozime and secretory IgA; caseins include $\alpha$-, $\beta$ - and $\kappa$-casein. Of note, caseins in human milk represent $13 \%$ of the total amount of pro- teins, thus representing the lowest casein concentration of any studied species. Mucins are incorporated into the membrane of the milk fat globule. 4

During the digestion process, most proteins are decomposed to free amino acids that are absorbed and utilized as building block for synthetizing new proteins in the body. However, it has to be taken into account that some nutritive proteins, that are partly or well absorbed, may exert biological functions. Furthermore it is now well acknowledged that some bioactive proteins are detected intact in infants stool. Taken all together, these data suggest that proteins are involved in the mechanisms responsible for the beneficial health effects associated with breastfeeding, such as the reduced risk of developing morbidities, particularly infections. ${ }^{12,13}$

Increasing evidence indicates that the quantity and the quality of protein in human milk play a crucial role in modulating infant growth and body composition. A high protein intake in infancy has actually been associated with an increased weight gain and risk of developing obesity later in life through the activation of the insulin-like growth factor-1 axis. ${ }^{14}$ De Luca et al. (2016) reported that the mature breast milk of obese mothers (body mass index $=34.3 \pm 3.9 \mathrm{~kg} / \mathrm{m}^{2}$ ), contained a higher amount of branchedchain amino acids $(+20 \%)$ and tyrosine $(+30 \%)$ compared to mature milk of non-obese mothers (body mass index $=21.6 \pm 1.4$ $\left.\mathrm{kg} / \mathrm{m}^{2}\right) .{ }^{15}$ Since high plasmatic concentrations of branched-chain amino acids appear to inhibit fatty free acids oxidation and, hence, to promote fat deposition, 16 these findings needs further clarification in terms of potential clinical implication, with regard to metabolic programming in breastfed infants.

\section{Non-protein nitrogen}

Non-protein nitrogen, including urea, creatinine, nucleotides, free amino acids and peptides, comprises $25 \%$ of the total amount of human milk nitrogen. Of note, nucleotides may be considered as conditionally essential nutrients. They play an important role in

\section{Protein $\mathrm{g} / 100 \mathrm{ml}$}

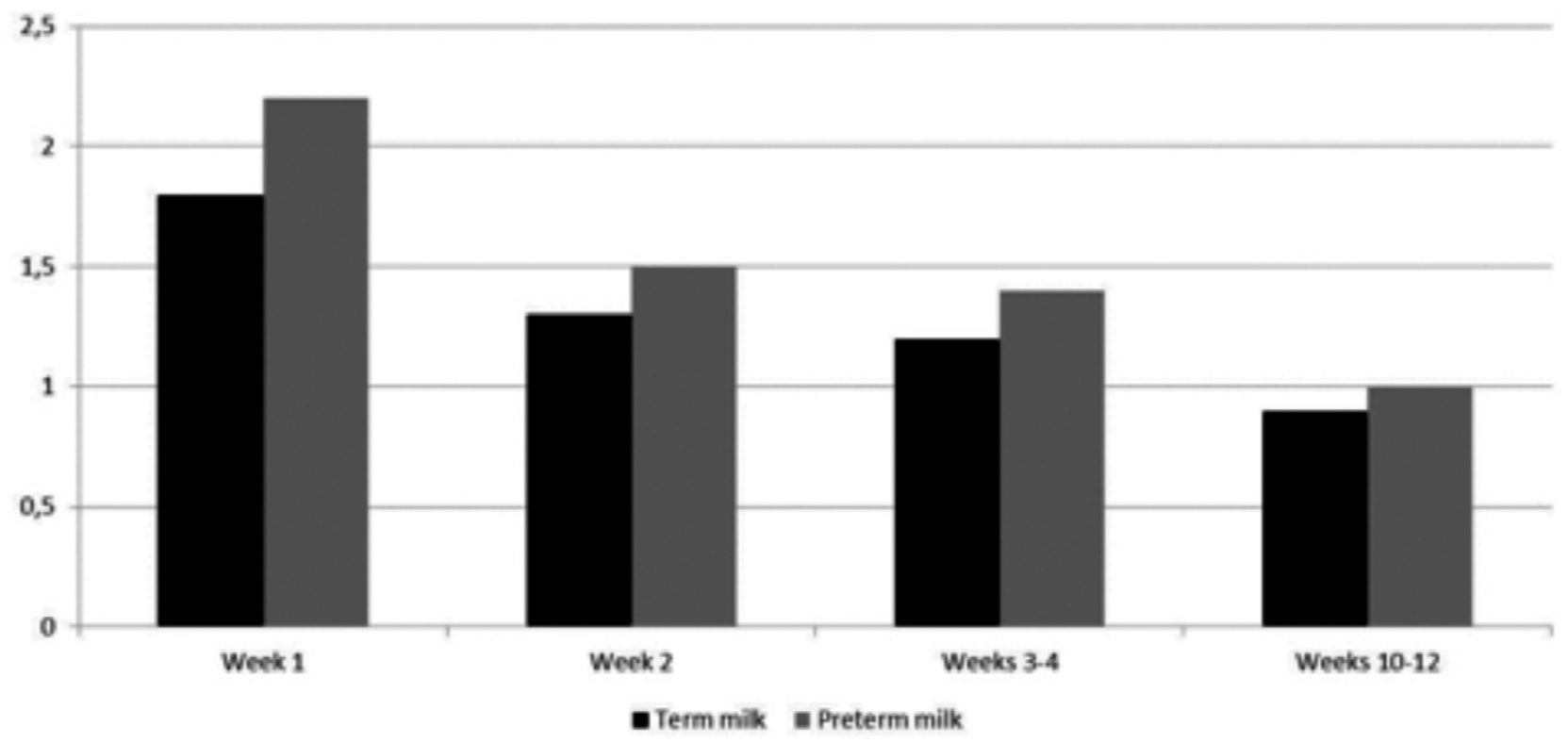

Figure 1. Protein content in preterm and term milk. 
several cellular functions, acting as metabolic modulator, modulating enzymatic activities and promoting the development and maturation of the gastrointestinal and immunological systems. ${ }^{4}$

\section{Carbohydrates}

The principal sugar of human milk is the disaccharide lactose. Its high concentration in human milk, $6.7 \mathrm{~g} / 100 \mathrm{~mL}$, which exceeds that of other species, reflects the high nutritional requirements of human brain. ${ }^{4}$ In addition, lactose represents an important source of galactose which, in turns, is important in promoting the development of the central nervous system. The other significant carbohydrates of human milk are the oligosaccharides (HMO), ranging between 1-10 $\mathrm{g} / \mathrm{L}$ in mature milk and $15-23 \mathrm{~g} / \mathrm{L}$ in colostrum. In mature milk $35-$ $50 \%$ of HMO are fucosylated, $12-14 \%$ are sialylated and $42-55 \%$ are nonfucosylated neutral, respectively. ${ }^{17}$ However, HMO specific composition is markedly affected by maternal genetic factors. ${ }^{11,18,19}$ Different structures of oligosaccharides depend on the presence of specific transferase enzymes expressed in the lactocytes. The two genes involved in shaping the specific composition are the Secretor and the Lewis Blood Group genes. It has been hypothesized that the variation in HMO composition among mothers may endorse human survival, as pathogens show different affinity for binding according to specific structures of oligosaccharides. ${ }^{9}$ Indeed, it has been demonstrated that infants fed milk from non secretor mothers are at higher risk for developing diarrhea. ${ }^{20}$ In experimental models it has been reported that only one type of HMO, that is disialyllacotse-Ntetraose (DSLNT), is protective against the risk of developing necrotizing enterocolitis (NEC). This finding further underlines that the protective role is actually strictly related to the specific HMO structures. ${ }^{21}$ Accordingly, Autran et al. (2017) have analysed the specific HMO content of 200 breastfeeding preterm mothers and reported that the DSLNT concentration was significantly lower in the milk fed to the infants that have developed NEC compared to that in the milk fed to the infants that had not developed NEC. 22

HMOs serve as prebiotics, acting as metabolic substrates for bacteria, thus enhancing the growth of beneficial bacteria, including Bifidobacteria and Bacteroides species. Furthermore HMO modulate several infant mucosal and systemic immune functions (Table 1). 17

HMO are also absorbed into the blood and have been reported to influence binding of monocyte, lymphocytes and neutrophils and formation of platelet-neutrophil complexes. It has also been speculated that they could exert some modulatory functions on the development of organs other than the gut. ${ }^{23}$

\section{Lipids}

Lipids represent the major sources of energy, contributing to the $44 \%$ of the total energy provided by human milk (Table 2 ). ${ }^{24}$

Furthermore they are an important source of essential nutrients such as polyunsaturated fatty acids (PUFA), lipid soluble vitamins, complex lipids and bioactive compounds. ${ }^{25}$ Lipids are present in the form of dispersed fat globules, comprising a core of non-polar lipids, mainly triacylglycerols, formed in the endoplasmic reticulum of the mammary epithelial cells. Upon the secretion from the endoplasmic reticulum into the cytosol, the core is first covered by an inner membrane deriving from the endoplasmic reticulum of the mammary alveolar cells. When these droplets are further excreted into the alveolar space, they are covered by an outer membrane deriving from the mammary alveolar cell plasma membrane. Hence, the milk fat globule membrane (MFGM) contains high amount of bioactive compounds, including glycerophospholipids, sphingolipids, sphingomyelin, glycolipids, cholesterol, glycosylated proteins. ${ }^{26}$ Accordingly, increasing attention has been focused on the potential biological functional effects of these components when added to infant formulas. Specifically, formula supplementation with MFGM has been reported to be associated with positive effects on neurocognitive development and immune function. ${ }^{27}$

Triacylglycerols represents $98 \%$ of human milk fat and their properties are largely affected by their fatty acid composition. Human milk contains more than 200 fatty acids, which are present in different concentrations. Milk lipids of European women are reported to be constituted by $35-40 \%$ of saturated fatty acids, 45 $50 \%$ of monounsaturated and $15 \%$ of polyunsaturated fatty acids. ${ }^{24}$

Palmitic acid provides the majority of saturated fatty acids. Of note, human milk palmitic acid is commonly found in the sn-2 position, which enhances its absorption and reduces calcium malabsorption.

The human milk content of fatty acids, particularly with regard to the long-chain polyunsaturated fatty acids (LCPUFAs), is largely affected by maternal diet. In the western world it has been recorded an increase in the omega 6/omega 3 ratio, that underlines a suboptimal intake of omega 3 associated with a higher consumption of omega- $6 .{ }^{9}$ About $75 \%$ of linoleic acid contained in human milk derives directly form dietary intake whereas $30 \%$ originates from maternal stores, being promptly transferred in the case of decreased dietary intake. However, persistent changes in dietary supply lead to modification of maternal stores. ${ }^{25,28}$

LCPUFAs exert several major biological effects, particularly on membrane functions, eicosaenoid and docosanoid production and related physiological processes, including growth and immune response. Human milk lipids, particularly medium chain monoglycerides, have been found to inactivate several pathogens in vitro, including Group B streptococcus, thus contributing to the protection against invasive infections. ${ }^{29}$ In addition, LCPUFAs provision with human milk positively affects retinal and brain cortical functional development in infants. ${ }^{30}$ While the majority of milk arachidonic acid derives from maternal stores, dietary docosahexaenoic acid (DHA) strictly correlates with milk content. ${ }^{31,32}$ In order to guarantee a daily DHA supply of $100 \mathrm{mg}$ to an exclusively breastfed infants, the breastfeeding mother should consume at least $200 \mathrm{mg}$ /day of DHA, required to achieve a milk DHA content of $0.3 \%$ of total fatty acids, which is advisable for meeting the infant's metabolic requirements. 33

Table 1. Potential mechanisms involved in human milk oligosaccharides modulation of infant mucosal and systemic immune functions. Reduction of intestinal crypt cell proliferation Increase of intestinal cell maturation and barrier function

Potential influence on globet cell function

Shaping of the epithelial immune gene expression either directly or indirectly through the influence on microbiota development

Inhibition of infections by either binding to the pathogen in the lumen or by inhibiting binding to cell-surface glycan receptors

Modulation of immune cell population and cytokine secretion 
Short chain fatty acids represent an important source of energy; furthermore they contribute to the gastrointestinal tract maturation.

\section{Other bioactive components}

Human milk is particularly rich in microRNAs, which are potentially involved in infant protection and development. MicroRNAs are small non-coding RNA molecules, that regulate gene expression at the post-transcriptional level, modulating several cell functions such as cell cycle, proliferation, differentiation, apoptosis, and immune response. ${ }^{34}$ Human milk has also been identified as the first probiotic food fed to the infants since it contains a diverse microbial community including $>200$ phylotypes. ${ }^{35}$ However, it has not been clearly elucidated how these bacteria reach the mammary gland and are finally incorporated into human milk. Studies suggest that an enteromammary pathway brings bacteria that are present in the maternal gut to the mammary gland via lymph and blood circulation. ${ }^{36}$ Resent research also indicates that the cellular fraction of human milk includes not only immune cells but also non immune ones, such as stem cells. The specific functional role associated with stem cells is not known; however, this finding suggests potential developmental benefits to the breast-fed infant. 35

Human milk contains also hormones that are acknowledged to modulate metabolism and body composition, such as insulin, leptin, adiponectin, ghrelin. Particularly, leptin milk concentration has been found to be directly correlated with maternal body mass index. 37,38

\section{Breastfeeding and associated outcomes}

Advances in research have clearly indicated that human milk offers both optimal nutrient supply and bioactive compounds that contribute to the short and long-term health benefits associated with breastfeeding. Remarkably, many health outcomes have been reported to be directly correlated with the duration of breastfeeding, suggesting a potential cumulative effect.

\section{Short-term health outcomes}

A systematic review published in 2016 indicates a strong protective effect of exclusive breastfeeding during the first six months of life against infectious diseases, with a $88 \%$ reduction of mortality in comparison to infants that have never been breastfed. $39,40 \mathrm{Of}$ note, in three studies conducted in low and medium income countries, the lack of breastfeeding has been associated with an increased risk of mortality. Specifically, males and females younger than six months, exhibited a 3.5 and 4.1 times increases in risk compared to any breastfeeding infants, respectively. 41 The dose dependent effect of breastfeeding is further supported by the finding that exclusively breastfeeding was associated with 48 and $78 \%$ reduction of risk of death when compared to partial and predominant breastfeeding, respectively. 40 Ever breastfeeding appears also to be protective against sudden infant death syndrome, being

Table 2. Mean macronutrients contribution to total energy intake of breastfed infants at 1 month of age.

$$
\text { Bnergy intake (\%) }
$$

\begin{tabular}{lc} 
Lipids & $44.5 \pm 5.2$ \\
Proteins & $8.4 \pm 1.0$ \\
\hline Carbohydrates & $43.9 \pm 5.8$
\end{tabular}

associated with a $36 \%$ [95\% confidence interval (CI) 19-49] reduction of its occurrence. 42

Compelling evidence indicates a major protective role of breastfeeding also with regard to the risk of diarrhea and respiratory infections, including hospitalization due to these diseases. Specifically, longer exposure to breastfeeding is associated with a relative risk of 0.69 (95\% CI 0.58-0.82) for developing diarrhea within the first five years of age and a $72 \%$ reduction of hospital admissions. ${ }^{43}$ About one third of the occurring lower respiratory diseases, including half of the hospitalizations due to their occurrence, could be prevented by breastfeeding. ${ }^{43} \mathrm{~A}$ reduced risk for developing otitis media is also reported in association with longer breastfeeding compared to a shorter one [odds ratio $(\mathrm{OR})=0.67 ; 95 \%$ CI $0.62-0.72]$; however, the protective effect is no more consistently detectable after two years of age. ${ }^{44}$ According to Victoria et al. (2016), there is no clear consistent evidence indicating a protective effect of breastfeeding towards either eczema or food allergy. 39 With regard to asthma, a $9 \%$ (95\% CI 2-15) reduction of the risk of its occurrence has been reported; however, when considering only the 16 studies with the most strict inclusion criteria and the 13 cohort studies, the positive association between breastfeeding and the reduced risk of asthma resulted not significant. 39

Furthermore a $68 \%$ (CI 95\% 60-75) reduction in the risk of malocclusion has been demonstrated if the infant is breastfed. 45 However, it has to be taken into account that breastfeeding for longer than one year and nocturnal feeding have been associated with an increased incidence of dental caries in deciduous teeth. It can be speculated that this effect may be due to inadequate oral hygiene after feeding. 46

\section{Long-term health outcomes}

Among the long term health outcomes, longer exposure to breastfeeding is associated with $35 \%(95 \%$ CI 14-51) reduction of the risk of developing diabetes type II. ${ }^{47}$ No significant evidence of a protective effect of breastfeeding towards hypertension and/or hypercholesterolemia has been demonstrated. ${ }^{47}$

As far as overweight/obesity are concerned, a meta-analysis including 113 studies demonstrated an association between $26 \%$ reduction of the risk of developing these conditions and longer breastfeeding. Remarkably, even when including only the 23 high quality studies, the positive association between breastfeeding and the $13 \%$ (95\% CI 6-19) reduced risk of overweight and obesity remained significant. 39,47

Amitay et al. (2015) have reported that the risk for developing leukemia was reduced by $19 \%$ in infants that were any breastfed compared to infants that had never been breastfed. 48

Consistent evidence indicates that breastfeeding is associated with positive cognitive outcome. Specifically, the intelligence quotient in ever breastfed /longer breastfed infants has been reported to be 3.4 (95\% CI 2.3-4.6) points higher than that of never breastfed/shorter breastfed infants. 49 Of note, the 16 studies included in the meta-analysis controlled for several potential confounding factors; furthermore, even when considering the nine studies that adjusted also for maternal intelligence quotient, breastfeeding remained associated with an increase in 2.6 points in the intelligent quotient.

\section{Health outcomes in preterm infants}

Human milk is recommended as the optimal nutrition not only for the full term infants but also for the preterm infants, particularly those born extremely preterm. ${ }^{1}$ Human milk consumption has 
been reported to significantly reduce complications associated with prematurity, including necrotizing enterocolitis, retinopathy of prematurity, bronchopolmunary dysplasia and late-onset sepsis. Furthermore human milk feeding promotes feeding tolerance with a reduced number of days needed to achieve the full enteral feeding. ${ }^{50,51}$ Long term benefits include reduced risk of re-hospitalization due to infectious diseases, particularly respiratory diseases, and promotion of both brain development and neurocognitive outcome. ${ }^{52,53}$ Recent research has also indicated a potential cumulative effect of human milk feeding on promotion of fat free mass deposition, which, in turns, appears to promote cognitive outcome. $54-56$

\section{Maternal health outcomes}

The meta-analysis conducted by Victoria et al., 2016 further underlined the well-known role of breastfeeding in birth spacing. Longer breastfeeding, and especially exclusive breastfeeding, were associated with longer periods of amenorrhoea. ${ }^{39}$ Compelling evidence demonstrates an inverse relationship between breastfeeding and breast cancer. Each increase in 12-month duration in lifetime breast feeding has been associated with a reduction of $4.3 \%$ (95\% CI 2.9-6.8) in the incidence of breast cancer. ${ }^{57}$ The meta-analysis by Victoria et al., 2016 confirmed the direction of this relationship and found a reduction of 7\% (95\% CI 3-11) in the risk of developing breast cancer with longer breastfeeding duration. 39 A 30\% (95\% CI 25-36) reduction in the incidence of ovarian cancer has also been associated with longer periods of breastfeeding. ${ }^{58}$ When considering only data deriving from studies with fine adjustment for parity and exclusion of nulliparous women, the risk of ovarian cancer was still significant although reduced to $18 \%$. Aune et al. (2014) conducted a meta-analysis, including six cohort studies, and found a protective effect of breastfeeding on type 2 diabetes $(\mathrm{OR}=0.68$; $95 \%$ CI 0.57 0.82). ${ }^{59}$ Few studies are available for the long-term association between nursing and adiposity. Mean body maternal index has been reported to be $1 \%$ lower for every 6 months increase in the lifetime breastfeeding duration. ${ }^{60}$ Although breastfeeding has been reported to be protective against depression, it can be argued that depression could actually affect breastfeeding. ${ }^{61}$

\section{Conclusions}

On the basis of the available evidence, human milk not only provides the perfectly adapted nutritional supply for the infant but also plays a crucial role in promoting healthy growth and development. Hence, protection, support and promotion of breastfeeding should be considered as a public health issue.

\section{References}

1. Johnston M, Landers S, Noble L, et al. Breastfeeding and the use of human milk. Section on breastfeeding. Pediatrics 2012; 129:e827-41.

2. WHO/UNICEF. Global strategy for infant and Young Child feeding. Geneva: World Health Organization; 2003.

3. Sharp JA, Modepalli V, Enjapoori AK, et al. Bioactive functions of milk proteins: a comparative genomics approach. J Mammary Gland Biol Neoplasia 2014;19:289-302.
4. Andreas NJ, Kampmann B, Mehring Le-Doare K. Human breast milk: a review on its composition and bioactivity. Early Hum Dev 2015;91:629-35.

5. Gidrewicz DA, Fenton TR. A systematic review and metaanalysis of the nutrient content of preterm and term breast milk. BMC Pediatr 2014;14:216.

6. Kader MM, Bahgat R, Aziz MT, et al. Lactation patterns in Egyptian women. II. Chemical composition of milk during the first year of lactation. J Biosoc Sci 1972;4:403-9.

7. Bravi F, Wiens F, Decarli A, et al. Impact of maternal nutrition on breast-milk composition: a systematic review. Am J Clin Nutr 2016;104:646-62.

8. Mäkelä J, Linderborg K, Niinikoski H, et al. Breast milk fatty acid composition differs between overweight and normal weight women: the STEPS Study. Eur J Nutr 2013;52:727-35.

9. Ballard O, Morrow AL. Human milk composition: nutrients and bioactive factors. Pediatr Clin North Am 2013;60:49-74.

10. Castellote C, Casillas R, Ramirez-Santana C, et al. Premature delivery influences the immunological composition of colostrum and transitional and mature human milk. J Nutr 2011;141:1181-7.

11. Smilowitz JT, Lebrilla CB, Mills DA, et al. Breast milk oligosaccharides: structure-function relationships in the neonate. Annu Rev Nutr 2014;34:143-69.

12. Haschke F, Haiden N, Thakkar SK. Nutritive and Bioactive Proteins in Breastmilk. Ann Nutr Metab 2016;69(Suppl. 2):17-26.

13. Lönnerdal B. Human milk: bioactive proteins/peptides and functional properties. Nestle Nutr Inst Workshop Ser 2016;86:97-107.

14. Luque V, Closa-Monasterolo R, Escribano J, Ferré N. Early programming by protein intake: the effect of protein on adiposity development and the growth and functionality of vital organs. Nutr Metab Insights 2016;8(Suppl.1):49-56.

15. De Luca A, Hankard R, Alexandre-Gouabau MC, et al. Higher concentrations of branched-chain amino acids in breast milk of obese mothers. Nutrition 2016;32:1295-8.

16. Kirchberg FF, Harder U, Weber M, et al. Dietary protein intake affects amino acid and acylcarnitine metabolism in infants aged 6 months. J Clin Endocrinol Metab 2015;100:149-58.

17. Donovan SM, Comstock SS. Human milk oligosaccharides influence neonatal mucosal and systemic immunity. Ann Nutr Metab 2016;69(Suppl.2):42-51.

18. Kunz C, Meyer C, Collado MC, et al. Influence of gestational age, secretor, and lewis blood group status on the oligosaccharide content of human milk. J Pediatr Gastroenterol Nutr 2017; 64:789-98.

19. Martín-Sosa S, Martín MJ, García-Pardo LA, Hueso P. Sialyloligosaccharides in human and bovine milk and in infant formulas: variations with the progression of lactation. J Dairy Sci 2003;86:52-9.

20. Lewis ZT, Totten SM, Smilowitz JT, et al. Maternal fucosyltransferase 2 status affects the gut bifidobacterial communities of breastfed infants. Microbiome 2015;3:13.

21. Jantscher-Krenn E, Zherebtsov M, Nissan C, et al. The human milk oligosaccharide disialyllacto-N-tetraose prevents necrotising enterocolitis in neonatal rats. Gut 2012;61:1417-25.

22. Autran CA, Kellman BP, Kim JH, et al. Human milk oligosaccharide composition predicts risk of necrotising enterocolitis in preterm infants. Gut 2017 (in press).

23. Dotz V, Rudloff S, Meyer C, et al. Metabolic fate of neutral human milk oligosaccharides in exclusively breast-fed infants. Mol Nutr Food Res 2015;59:355-64.

24. Grote V, Verduci E, Scaglioni S, et al. Breast milk composition 
and infant nutrient intakes during the first 12 months of life. Eur J Clin Nutr 2016;70:250-6.

25. Koletzko B. Human Milk Lipids. Ann Nutr Metab 2016;69 (Suppl.2):28-40.

26. Contarini G, Povolo M. Phospholipids in milk fat: composition, biological and technological significance, and analytical strategies. Int J Mol Sci 2013;14:2808-31.

27. Timby N, Domellöf M, Lönnerdal B, Hernell O. supplementation of infant formula with bovine milk fat globule membranes. Adv Nutr 2017;8:351-5.

28. Demmelmair H, Baumheuer M, Koletzko B, et al. Metabolism of U13C-labeled linoleic acid in lactating women. J Lipid Res 1998;39:1389-96.

29. Isaacs CE, Litov RE, Thormar H. Antimicrobial activity of lipids added to human milk, infant formula, and bovine milk. J Nutr Biochem 1995;6:362-6.

30. Uauy R, Mena P, Rojas C. Essential fatty acids in early life: structural and functional role. Proc Nutr Soc 2000;59:3-15.

31. Del Prado M, Villalpando S, Elizondo A, et al. Contribution of dietary and newly formed arachidonic acid to human milk lipids in women eating a low-fat diet. Am J Clin Nutr 2001;74: 242-7.

32. Fidler N, Sauerwald T, Pohl A, et al. Docosahexaenoic acid transfer into human milk after dietary supplementation: a randomized clinical trial. J Lipid Res 2000;41:1376-83.

33. Koletzko B, Lien E, Agostoni C, et al. The roles of long-chain polyunsaturated fatty acids in pregnancy, lactation and infancy: review of current knowledge and consensus recommendations. J Perinat Med 2008;36:5-14.

34. Alsaweed M, Lai CT, Hartmann PE, et al. Human milk cells and lipids conserve numerous known and novel miRNAs, some of which are differentially expressed during Lactation. PLoS One 2016;11:e0152610.

35. Bode L, McGuire M, Rodriguez JM, et al. It's alive: microbes and cells in human milk and their potential benefits to mother and infant. Adv Nutr 2014;5:571-3.

36. Funkhouser LJ, Bordenstein SR. Mom knows best: the universality of maternal microbial transmission. PLoS Biol 2013;11: e1001631.

37. Demmelmair H, Koletzko B. variation of metabolite and hormone contents in human milk. Clin Perinatol 2017;44:151-64.

38. Fields DA, Schneider CR, Pavela G. A narrative review of the associations between six bioactive components in breast milk and infant adiposity. Obesity 2016;24:1213-21.

39. Victora CG, Bahl R, Barros AJ, et al. Breastfeeding in the $21 \mathrm{st}$ century: epidemiology, mechanisms, and lifelong effect. Lancet 2016;387:475-90.

40. Sankar MJ, Sinha B, Chowdhury R, et al. Optimal breastfeeding practices and infant and child mortality. A systematic review and meta-analysis. Acta Paediatr 2015;104:3-13.

41. WHO Collaborative Study Team on the Role of Breastfeeding on the prevention of Infant Mortality. Effect of breastfeeding on infant and child mortality due to infectious diseases in less developed countries: a pooled analysis. Lancet 2000;355:451-5.

42. Ip S, Chung M, Raman G, et al. Breastfeeding and maternal and infant health outcomes in developed countries. Rockville, MD, USA: Agency for Healthcare Research and Quality; 2007.

43. Horta BL, Victora CG. Short-term effects of breastfeeding: a systematic review of the benefits of breastfeeding on diarhoea and pneumonia mortality. Geneva: World Health Organization; 2013.
44. Bowatte G, Tham R, Allen KJ, et al. Breastfeeding and childhood acute otitis media: a systematic review and meta-analysis. Acta Paediatr Suppl 2015;104:85-95.

45. Peres KG, Cascaes AM, Nascimento GG, Victora CG. Effect of breastfeeding on malocclusions: a systematic review and meta-analysis. Acta Paediatr Suppl 2015;104:54-61.

46. Tham R, Bowatte G, Dharmage SC, et al. Breastfeeding and the risk of dental caries: a systematic review and meta-analysis. Acta Paediatr Suppl 2015;104:62-84.

47. Horta BL, de Mola CL, Victora CG. Long-term consequences of breastfeeding on cholesterol, obesity, systolic blood pressure, and type-2 diabetes: systematic review and meta-analysis. Acta Paediatr Suppl 2015;104:30-37.

48. Amitay EL, Keinan-Boker L. Breastfeeding and childhood leukemia incidence: a meta-analysis and systematic review. JAMA Pediatr 2015;169:e151025.

49. Horta BL, de Mola CL, Victora CG. Breastfeeding and intelligence: systematic review and meta-analysis. Acta Paediatr Suppl 2015;104:14-9.

50. Menon G, Williams TC. Human milk for preterm infants: why, what, when and how? Arch Dis Child Fetal Neonatal Ed 2013;98:F559-62.

51. Radmacher PG, Adamkin DH. Fortification of human milk for preterm infants. Semin Fetal Neonatal Med 2017;22:30-5.

52. Vohr BR, Poindexter BB, Dusick AM, et al. Persistent beneficial effects of breast milk ingested in the neonatal intensive care unit on outcomes of extremely low birth weight infants at 30 months of age. Pediatrics 2007;120:e953-9.

53. Lechner BE. Neurodevelopmental outcomes of preterm infants fed human milk. A systematic review. Clin Perinatol 2017;44: 69-83.

54. Larcade J, Pradat P, Buffin R, et al. Estimation of fat-free mass at discharge in preterm infants fed with optimized feeding regimen. J Pediatr Gastroenterol Nutr 2017;64:115-8.

55. Giannì ML, Consonni D, Liotto N, et al. Does human milk modulate body composition in late preterm infants at term-corrected age? Nutrients 2016;23:8.

56. Ramel SE, Gray HL, Christiansen E, et al. Greater early gains in fat-free mass, but not fat mass, are associated with improved neurodevelopment at 1 year corrected age for prematurity in very low birth weight preterm infants. J Pediatr 2016;173:108-15.

57. Collaborative Group on Hormonal Factors in Breast Cancer. Breast cancer and breastfeeding: collaborative reanalysis of individual data from 47 epidemiological studies in 30 countries, including 50302 women with breast cancer and 96973 women without the disease. Lancet 2002;360:187-95.

58. Chowdhury R, Sinha B, Sankar MJ, et al. Breastfeeding and maternal health outcomes: a systematic review and metaanalysis. Acta Paediatr Suppl 2015;104:96-113.

59. Aune D, Norat T, Romundstad P, Vatten LJ. Breastfeeding and the maternal risk of type 2 diabetes: a systematic review and dose-response meta-analysis of cohort studies. Nutr Metab Cardiovasc Dis 2014;24:107-15.

60. Bobrow KL, Quigley MA, Green J, et al. Persistent effects of women's parity and breastfeeding patterns on their body mass index: results from the Million Women Study. Int J Obes 2013; 37:712-17.

61. Dias CC, Figueiredo B. Breastfeeding and depression: a systematic review of the literature. J Affect Disord 2015;171:142-54. 